\title{
A Game Theoretic Approach to assessing Additive Manufacturing as a Strategic Choice
}

\author{
Mudassar Ahmed \\ Institute for Manufacturing \\ University of Cambridge \\ ma603@cam.ac.uk
}

\author{
Keith Jin Deng Chan \\ Faculty of Economics \\ University of Cambridge \\ jdkc2@cam.ac.uk
}

\author{
Duncan McFarlane \\ Institute for Manufacturing \\ University of Cambridge \\ dcm@eng.cam.ac.uk
}

\begin{abstract}
This paper studies the impact of demand uncertainty and competition on a firms technology selection decision between Additive Manufacturing (AM) and conventional toolbased Dedicated Manufacturing (DM). The paper uses the gametheoretic approach presented in [57], and extends the model from 2 to $n$ players making three sequential decisions under demand uncertainty: (i) choice of technology, (ii) capacity investment, and (iii) production quantities. We find that the conventional wisdom of choosing between flexible manufacturing technology and dedicated tool-based technology holds in the case of AM given there is no demand uncertainty. However, once we take the demand uncertainty into account, a game-theoretical analysis is essential to examine the profitability of switching manufacturing technologies to AM. The research will contribute towards a future empirical study and will help develop an industrially accessible decision support tool for the adoption of $A M$ in a production environment.
\end{abstract}

Index Terms-Additive Manufacturing; 3d Printing; Dedicated Manufacturing; Tool-based Manufacturing; Game theory; Technology Choice; Technology Selection; Decision Support tool

\section{INTRODUCTION}

The global manufacturing sector is undergoing a tumultuous change. While the20th century was about hundreds of products selling millions of copies, the 21 st century isabout millions of products selling hundreds of copies.Customers and markets are demanding increased product customisation, greater product variety and frequent changes in batch sizes. As an industry, manufacturing patterns are shifting awayfrom identical mass production paradigm introduced by Henry Ford towards more customised solutions.

There is an enormous pressure on all manufacturing businesses to be more responsive in the way they operate and respond to disruptions. Disruptions are those sources of change, which occur independently of the systems intentions [1]. These disruptions are categorised into three broad areas [2]:

- Upstream (Supplier): Problems associated with materials quality, delivery delays, incorrect deliveries

- Internal (Manufacturing System): Machine breakdowns, production rate, quality, labour, operator errors.

- Downstream (Customer): Customisation requests, rush orders due to demand variations (activity, seasonality, marketing, competitors activity), changes to orders (quantity, mix, due date); forecasting errors
Responsiveness is the ability of the manufacturing system to respond to customer requests within a minimal timeframe. The debate about the responsiveness of manufacturing operations has been going on for more than two decades, with its origins in the concept of time compression or time based competition [3]-[4], and it was later adopted by a range of companies and academic researchers [5]-[6]. These approaches are then categorised into three dimensions of responsiveness [7]:

- Product: Customisation point(s), external variety (postconfiguration), internal variety (pre-configuration)

- Process: Production lead times, decoupling point(s)

- Volume: Variability in demand, customer expectations

The ability to mitigate the impact of disruptions are related to the development of the above identified responsiveness capabilities. Resilience is a similar but a broader concept. It is the ability to withstand such unexpected disruptions and operate smoothly in a volatile environment [8]. In a recent paper, [9] has identified four distinct stages in building resilience dealing with disruptions, namely: (i) Awareness, (ii) Preparation, (iii) Management and (iv) Response. Two strategies widely used to mitigate or withstand these disruptions are through (i) redundancies, and (ii) flexibilities [10]. Redundancy entails keeping some resources in reserve to respond to disruption, while flexibility entails restructuring previously existing capacity [11].

Many business continuity plans are based on increasing redundancy in several facets of the companies operations. The most common forms of redundancy are safety stock (storage buffers), multiple (second) suppliers, and deliberately low capacity utilisation rates [10]. Traditionally, the production lines are equipped with storage zones (buffers) in different locations along the system to provide protection against any disruptions by employing redundancy. Allocation of buffer size and location in production systems is an important optimisation problem faced by manufacturing system designers. An analytical approach to identify a trade-off between small and large inventory buffers and their locations to respond to disruptions is discussed in [8]. Such investments, however, can only go a limited way 
towards addressing disruptions, as they present a cost to the company with a return that can only be realized in case of a major disruption. Investing in flexibility, however, yields many additional benefits for day-to-day operations [10].

Flexibility in the manufacturing context is generally considered as an adaptive response to environmental uncertainty [12]-[13]. The ability of a system to adapt to changing external and internal influences has been recognised as a source of competitive advantage [14]-[15], which was widely promoted in the 1990s [16]-[17]. It has been employed as a weapon to deter new entrants [18]. As the importance of the ability to adapt to changing market environments grew, the debate on flexibility provided the theoretical origin for the concepts of responsiveness [19]. Although the results of increased flexibility are difficult to measure with traditional accounting and risk management tools, investment in flexibility can be justified in terms of the increased sales, reduced costs and increased competitive advantage that companies can enjoy by developing flexible operations [10].

The various forms of machine and subsystem flexibilities found within a production system often represent means of responding to disruptions [20]. The adoption of flexible manufacturing systems (FMS) has had significant implications for manufacturers and market structure [21]-[22]. FMS can flexibly produce a variety of outcomes using the same (manufacturing) resources [13]. The strategies discussed in the literature include late configuration, modularity, postponement and standardisation [23]-[24]. More recently, research has highlighted the potential of additive manufacturing (AM) technology to spark a new industrial revolution by extending the features of conventional FMS technology [25]-[29]. AM refers to the process of joining materials to make objects from 3D model data, usually layer upon layer [30]. Colloquially, $\mathrm{AM}$ is often referred to as $3 \mathrm{D}$ printing [31]

The main benefit of AM technology is that it enables the flexible production of customised products without cost penalties in manufacturing. AM does so by directly transforming 3D data into physical parts, without any need for molds or tools. Moreover, the layer-by-layer manufacturing can consolidate and produce functionally integrated parts in a single step, hence cutting down the need for assembly. Thus, AM technology significantly affects the costs of flexibility, capital costs, personalisation, and marginal production costs [25], [32]-[33]. The opportunities of AM, however, come with a number of technical and economic barriers that preclude its widespread use for commercial applications. These include a high machine cost, material cost, materials choices, dimensional accuracy, surface roughness, support design and removal, low mechanical properties, building time for large size component [25], [34]-[38]. Given these trade-offs, our paper aims to offer support in evaluating AM as a profitable means for responding to disruptions before making technology investment decision.

The remainder of the paper is structured as follows. Next section provides the review of technology selection techniques. Section 3 describes a simple extension of an existing game model in the literature. Section 4 solves this technology game under appropriate assumptions. The analysis and three propositions are outlined in section 5 and Section 6 concludes the paper.

\section{LiteratURE REVIEW}

The literature on the issue of selecting manufacturing technology in production systems is wide and varied. The implications of new technological advancements (e.g., CAD, $\mathrm{CNC}$, automation etc.) have been discussed by researchers in utilising flexibility as a strategic pillar for the generation of profit [13], [22]. These investment trade-offs have also been investigated in other contexts [13], [21]. We focus mainly on systems utilising AM as a profitable means for mitigating the effects of downstream disruptions.

At a firm level, the impact of manufacturing technology can be assessed using one of the most cited model for modern manufacturing that offers a theoretical foundation to perform a pay-off analysis [39]. The authors in [34] systematically adopt this model for AM technology. Their analysis reaffirms the intuition about the positive impact of AM on the profit of a monopolist firm i.e. premium priced customised products at no cost penalties can increase profits as long as the processing time, marginal costs of production, and defective batch probability are not high.

At a market structure level, two approaches with a formal analytical model have been used to study the impact of conventional FMS that incorporate both manufacturing flexibility and product differentiation. They are (i) product attribute address models, and (ii) game-theoretic models for technology choice. These models have primarily been used to assess the implication of technology choice (between dedicated and conventional FMS) [44]-[46], [57]-[59], however, given AM offers more than a conventional FMS in terms of both enhanced manufacturing flexibility and product differentiation [34], we therefore believe that both product attributes and game theoretic models can be adopted to discuss the effects of AM technology, as explained below. It should be noted, however, that the focus of our study is on the latter game-theoretic model approach.

Within the first product attributes address model approach, the scholars have examined the impact of (conventional) FMS on market structure. [44] models this effect by setting modification costs proportional to the distance between the base product and the variant, and conclude that flexibility in production systems promotes the concentration in market through pre-emption and mergers. The authors in [45] discuss the strategic aspects of the choice of technology. They 
found that firm can discriminate on prices as the price of one product variant can be independently adjusted without affecting other variants, thus suggesting that flexibility can precisely be used to deter entry by locally lowering prices as and when there is a threat of entry. [34] have adopted the product attributes models in [44] in light of the four key principles they identified for the production with AM technology. The authors have concluded that unlike the case of conventional FMS in [44], the incentives for mergers (given a firm with AM enters the market) are also reduced as AM leads to lower market prices. The authors in [34] also applied the AM characteristics to the model proposed in [45], and concluded that in the case of higher AM penetration, the incumbents no longer has the same strategic advantage (as in the case of conventional FMS) for deterring market entry by locally cutting down prices.

For the second approach of game-theoretic models, the scholars have assessed the choice of dedicated versus (conventional) flexible manufacturing technology. The literature relevant to the game-theoretic approaches can be categorised into two major streams, as is also done by [57]: the first exploring flexibility as a hedge against demand uncertainty, and the second studying flexibility as a strategic weapon under competition.

The first stream within game theoretic approaches consider the investment choice between dedicated capacity and the (conventional) flexible capacity in the absence of competition. It analyses the trade-off between the ability of (conventional) flexible manufacturing systems to hedge against demand uncertainty (by manufacturing a range of products) against the higher cost of flexibility. Without competition, invariably all papers consider a monopolistic firm. For instance, [18] assesses the monopolists rationale in a sequential game between the monopolistic incumbent and a potential entrant where both have the choice of adopting either flexible or dedicated manufacturing technology. The authors identify a zone of strategic flexibility, whereby they treat FMS as a strategic choice for firms to deter market entry and conclude that under uncertain consumer preferences, an incumbent threatened by new market entrants is more likely to install FMS than an unchallenged monopolist. More recently, again for a sequential game, [58] shows that for a not so uncertain environment, it is more profitable for the incumbent to invest in dedicated production line as a means for deterring an entrant. A range of authors [46]-[51] have looked into technology choice with product prices determined by an exogenous demand curve. [46] models a two-stage game and conclude that the technology investment decision between dedicated and (conventional) flexible manufacturing comes down to the cost differential. [47] studies the notion of total flexibility against partial flexibility by using a concept of chaining. [48] develops a similar model and shows that flexibility is beneficial even with perfect positive correlation if product margins are different. [52] let the firms set their product prices and compare the benefits of investment in flexible against two dedicated technologies under the assumption that investment costs for the two technologies are the same. They find that the flexible capacity and expected profits are increasing in demand variance, and that positive correlation increases capacity investment while decreasing expected profits.

The authors in the second stream of literature within game theory approaches for technology-choice look at the strategic value of flexibility in the absence of demand uncertainty. One of the first papers to explore technology adoption under competition is that of [53], who studies a differential game between two firms that can acquire new technology to reduce unit operating cost for a single product (hence, FMS or AM is outside of the scope of this work). [54]-[55] model two firms competing with each other in a two-stage game. First, firms decide on their investment in either flexible (can enter two markets) or dedicated manufacturing technology (can enter one market only). Second, firms choose their output volume for two differentiated product markets. The fixed costs for investments in FMS are always higher than or equal to those of dedicated manufacturing technology. The firms are therefore in a Prisoners dilemma, and hence these papers show that flexible technology is detrimental to both firms. [59] however argues that if the two products are not substitutes as is assumed in [54]-[55], and are in fact sufficiently complementary, and given the investment cost for flexible technology is not too large, then both firms investing flexible technology will benefit themselves simultaneously and hence prisoner's dilemma-like situation does not occur.

[57] combines the two streams of game-theoretical approaches by incorporating both competition and demand uncertainty in the model to study strategic technology choice between dedicated and conventional flexible manufacturing system. Unlike [56] which requires a firm to possess flexible technology to enter a second market, [57] relaxes this assumption. This implies that flexibility does not inherently fuel competition, and hence prices are no longer the lowest when both firms choose flexible technology. [57] then demonstrates the effects of demand uncertainty, market size, product substitutability, and cost on the equilibrium outcomes under simultaneous move in technology choices. While [57] shows the possibility of all technology choice combinations in equilibrium, [58] adopts an incumbent-entrant sequential structure and shows that the equilibrium where both firms invest in dedicated technology ceases to exist. With a view to focus on enhanced flexibility and product differentiation offered by AM, we adopt the model presented in [57] to study the technology choice between AM and dedicated manufacturing. Alongside many common features between $\mathrm{AM}$ and conventional FMS, nevertheless, we note that AM has its differences from conventional FMS as noted by [34] which include the higher marginal cost of AM and lower upfront and fixed cost (set up cost, switching cost etc.) amongst others. 
On top of the theoretical studies reviewed above, numerous scholars have conducted empirical studies, and looked into the cost models to assess the economic viability of additive manufacturing against conventional manufacturing [40]-[42]. The two models that receive significant attention are: (i) Hopkinson and Dickens [40], and (ii) Ruffo et al. [41]. [40] calculates the average cost per part using AM by dividing the total cost by the total number of parts manufactured in a year. The authors break the cost into machine costs, labour costs, and material costs amongst other (negligible) costs [42]. They calculate the costs for a lever and compare it against the injection modelling cost, and find a threshold value under which AM is a more economically viable technology. [41] however, use an activity based cost model. In their model, the total cost of a build (C), is the sum of raw material costs (price $/ \mathrm{kg}^{*}$ mass) and indirect costs (rate*build time). The cost per part is calculated as the total cost of a build (C) divided by the number of parts in the build. While producing the same lever as produced in [40], [41] indicate that the time and material used are the main variables in the costing model. Their model has a jagged saw tooth shape to it, which is due to the impact of a new line, layer, or build. Each time one of these is added, average costs increase irregularly from raw material consumption and process time. [43] uses the above model to compare the per-assembly processing cost for a landing gear assembly, and finds that for production runs of less than 42 units, AM was more cost effective than the conventional die-casting manufacturing.

These approaches seem to use the cost model without taking into account the demand they can capture, and by extension, the potential revenue, which is crucial for realising the profit in making technology investment decisions. On the other hand, a game-theoretical approach provides explicit account on how the market share of each firm also depends endogenously on the technology profile of the industry, which improves assessment on the net benefit of switching technology. This justifies our choice of using game theory for assessing the technology investment decision in the context of AM.

For the reasons outlined above, we take the model initially developed by [57] to assess AM as a strategic choice. We extend the model from 2- player to n-player to accommodate industry specific features such as technology penetration rate, and how the incentive for switching to AM technologies evolves at different stages of technology adoption in the market. This enriches the model in [57], and paves the way for our forthcoming empirical study.

\section{THE MODEL}

The model is an extension of [57], so we would only describe its key elements here without repeating the justification of each assumption. We adopt [57] as the base model because it captures the decision between two production technologies which differ in flexibility against actualized demand; we extend [57] from 2 players to $n$ players to capture the There are $n$ firms manufacturing two products (indexed by 1 and 2) and engaging in competition with one another in both markets. We assume that the firms are risk neutral and maximize expected profits. In the first stage, each firm simultaneously chooses to invest in either dedicated manufacturing technology (d) or additive manufacturing technology (a).

Depending on the technological choices, $2^{n}$ subgames can potentially emerge. In the second stage, each firm $i$ with additive manufacturing technology invests in a single production capacity $K_{i}^{a}$ with per unit cost $c^{a}$, whereas each firm $j$ with dedicated manufacturing technology invests in its production capacity for each of the two products $K_{1 j}^{d}$ and $K_{2 j}^{d}$ with per unit cost $c^{d}$. We assume the capacity costs to be homogeneous across firms for simplicity. This can be justified by assuming reasonable spread of technological knowledge within the industry.

In the third stage, demands for both products $A_{1}$ and $A_{2}$ are realized. Each firm $i$ chooses its production quantity $q_{i y}$ for each product $y=1,2$. Let $Q_{y}:=\sum_{i=1}^{n} q_{i y}$ be the total quantity of product $y$ put on the market by the $n$ firms. The inverse demand function for product $y$ is then $P_{y}\left(Q_{y}, Q_{3-y}\right)=A_{y}-Q_{y}-\beta Q_{3-y}$, where $\beta \in(-1,1)$ is the product substitutability parameter. For $y=1,2$, denote the mean of $A_{y}$ by $\mu_{y}$ interpreted as expected market size, and let $\sigma_{T}^{2}:=\operatorname{Var}\left(A_{1}-A_{2}\right)$ be the total demand uncertainty facing each firm.

\section{SOlVING THE GAME}

We adopt the solution concept of subgame-perfect equilibrium (SPE).

Proposition 1 Fix a subgame in the first stage where $m$ firms have adopted additive manufacturing technology, and $n-m$ firms have adopted dedicated manufacturing technology, for $m=0,1, \ldots, n$. In the unique SPE of this subgame, the expected profit of any firm with dedicated manufacturing technology is

$$
E \pi^{d}(m)=\frac{\left(\mu_{1}+\mu_{2}+2 m c^{a}-2(m+1) c^{d}\right)^{2}}{2(n+1)^{2}(1+\beta)}+\frac{\left(\mu_{1}-\mu_{2}\right)^{2}}{2(n+1)^{2}(1-\beta)}
$$

Meanwhile, the expected profit of any firm with additive manufacturing technology is

$$
\begin{aligned}
E \pi^{a}(m)=\frac{\left(\mu_{1}+\mu_{2}+2(n-m) c^{d}-2(n-m+1) c^{a}\right)^{2}}{2(n+1)^{2}(1+\beta)} \\
+\frac{\left(\mu_{1}-\mu_{2}\right)^{2}}{2(n+1)^{2}(1-\beta)}+\frac{\sigma_{T}^{2}}{2(m+1)^{2}(1-\beta)}
\end{aligned}
$$

We start from the third stage. Fix a subgame with firm $1,2, \ldots, m$ having adopted additive manufacturing technology, and all other firms dedicated manufacturing technology, without loss of generality. As in [57], we assume each 
firm produces to capacity, and each additive firm always manufactures both products. Given capacities $\left(K_{j}^{a}\right)_{j=1}^{m}$ and $\left(K_{1 j}^{d}, K_{2 j}^{d}\right)_{j=m+1}^{n}$, the realized demand $A_{1}$ and $A_{2}$, the objective of additive firm $i$ is to choose $\left(q_{1 i}^{a}, q_{2 i}^{a}\right)$ to maximize

$$
\begin{aligned}
\pi_{i}^{a}=\sum_{y=1}^{2}\left[A_{y}-\left(\sum_{h=1}^{m} q_{y h}^{a}\right.\right. & \left.+\sum_{h=m+1}^{n} K_{y h}^{d}\right) \\
& \left.-\beta\left(\sum_{h=1}^{m} q_{3-y, h}^{a}+\sum_{h=m+1}^{n} K_{3-y, h}^{d}\right)\right] q_{y i}^{a}
\end{aligned}
$$

under the constraint $q_{1 i}^{a}+q_{2 i}^{a} \leq K_{i}^{a}$. For $y=1,2$, this yields for each addition firm $i$ :

$$
q_{y i}^{a}=\frac{A_{y}-A_{3-y}+(1-\beta) \sum_{h=m+1}^{n}\left(K_{3-y, h}^{d}-K_{y h}^{d}\right)}{2(m+1)(1-\beta)}+\frac{K_{i}^{a}}{2}
$$

Next we proceed to the second stage. With this continuation response function derived above, the objective of additive firm $i$ is to choose $K_{i}^{a}$ to maximize

$$
\begin{aligned}
E \pi_{i}^{a}=E \sum_{y=1}^{2}\left[A_{y}\right. & -\left(\sum_{h=1}^{m} q_{y h}^{a}+\sum_{h=m+1}^{n} K_{y h}^{d}\right) \\
& \left.-\beta\left(\sum_{h=1}^{m} q_{3-y, h}^{a}+\sum_{h=m+1}^{n} K_{3-y, h}^{d}\right)\right] q_{y i}^{a}-c^{a} K_{i}^{a}
\end{aligned}
$$

First order condition yields for each additive firm $i$ :

$$
K_{i}^{a}=\frac{\mu_{1}+\mu_{2}-(1+\beta) \sum_{h=m+1}^{n}\left(K_{1 h}^{d}+K_{2 h}^{d}\right)-2 c^{a}}{(m+1)(1+\beta)}
$$

Meanwhile, the objective of dedicated firm $j$ is to choose $\left(K_{1 j}^{d}, K_{2 j}^{d}\right)$ to maximize

$$
\begin{aligned}
E \pi_{j}^{d}= & E \sum_{y=1}^{2}\left[A_{y}-\left(\sum_{h=1}^{m} q_{y h}^{a}+\sum_{h=m+1}^{n} K_{y h}^{d}\right)\right. \\
& \left.-\beta\left(\sum_{h=1}^{m} q_{3-y, h}^{a}+\sum_{h=m+1}^{n} K_{3-y, h}^{d}\right)\right] K_{y j}^{d}-c^{d}\left(K_{1 j}^{d}+K_{2 j}^{d}\right)
\end{aligned}
$$

With the expression of $\left(K_{i}^{a}\right)_{i=1}^{m}$ above, this yields for $y=1,2$ and each dedicated firm $j$ :

$$
K_{y j}^{d}(m)=\frac{\mu_{y}-\beta \mu_{3-y}+(1-\beta)\left(m c^{a}-(m+1) c^{d}\right)}{(n+1)\left(1-\beta^{2}\right)}
$$

Apply this back to the expression of $K_{i}^{a}$, we obtain

$$
K_{i}^{a}(m)=\frac{\mu_{1}+\mu_{2}+2(n-m) c^{d}-2(n-m+1) c^{a}}{(n+1)(1+\beta)}
$$

Substitute the above to the expected profit function of dedicated firms and additive firms yields the expressions in Proposition 1.

[57] has obtained the profit functions for the special case $n=2$. However, their coefficient for $\left(\mu_{1}-\mu_{2}\right)^{2}$ seems incorrect, which potentially affects their subsequent claim regarding the threshold of marginal cost of dedicated manufacturing above which switching to the flexible manufacturing technology becomes profitable. Other than that, the market size effect, substitutability effect, and stochastic effect they have identified remain valid in our extension.

\section{ANALYSIS}

Define the function $\Omega(m):=E \pi^{a}(m+1)-E \pi^{d}(m)$, which can be interpreted as the deviation profit of any dedicated firm for switching to additive manufacturing technology when $m$ other firms have adopted additive manufacturing technology. By definition and Proposition 1, we have

$$
\begin{aligned}
\Omega(m)= & \frac{2 n\left(c^{d}-c^{a}\right)}{(n+1)^{2}(1+\beta)}\left(\mu_{1}+\mu_{2}+(n-2 m-2) c^{d}-(n-2 m) c^{a}\right) \\
& +\frac{\sigma_{T}^{2}}{2(m+2)^{2}(1-\beta)}
\end{aligned}
$$

Proposition 2 The deviation profit $\Omega(m)$ of switching from dedicated manufacturing technology to additive manufacturing technology, when $m$ other firms are already adopting the latter, is decreasing in $m$.

This is straightforward because for $m<n$,

$\Omega(m+1)-\Omega(m)=-\frac{4 n\left(c^{d}-c^{a}\right)^{2}}{(n+1)^{2}(1+\beta)}-\frac{(2 m+5) \sigma_{T}^{2}}{2(m+2)^{2}(m+3)^{2}(1-\beta)}$

The above expression is obviously negative.

Proposition 2 implies that if a dedicated firm does not switch to additive manufacturing technology now, its deviation profit will only be lower in the future when other dedicated firms have made the switch. Hence, if a dedicated firm believes the market environment and production cost structure to be relatively stationary in the future, and the switch to additive manufacturing technology is unilateral, then it should switch now instead of waiting for its competitors, unless switching is not profitable to begin with.

Proposition 3 Suppose demand uncertainty $\sigma_{T}^{2}$ is zero. Then regardless of how many competitors are adopting additive manufacturing technology, it is profitable for a firm to switch from dedicated manufacturing technology to additive manufacturing technology if and only if the marginal cost of the former is higher.

This is equivalent to claiming $\Omega(m)>0$ if and only if $c^{a}<c^{d}$ for all $m$ given $\sigma_{T}^{2}=0$. From the proof of Proposition 1, we know

$$
\begin{aligned}
K_{1 j}^{d}(m)+K_{2 j}^{d}(m) & +K_{i}^{a}(m+1) \\
& =\frac{\mu_{1}+\mu_{2}+(n-2 m-2) c^{d}-(n-2 m) c^{a}}{0.5(n+1)(1+\beta)}
\end{aligned}
$$

But then $\mu_{1}+\mu_{2}+(n-2 m-2) c^{d}-(n-2 m) c^{a}$ must be positive.

Proposition 3 shows the conventional wisdom of choosing between dedicated and additive manufacturing technology is correct under the implicit assumption of zero demand uncertainty. Now we will study the realistic case of nonzero demand uncertainty, which justifies the value of gametheoretic analysis on top of the conventional decision rule. 
Proposition 4 Suppose demand uncertainty $\sigma_{T}^{2}$ is nonzero. Then it is profitable for a firm to switch from dedicated manufacturing technology to additive manufacturing technology if and only if the marginal cost of the latter is lower than some threshold $\Phi(m)$, which is greater than the dedicated manufacturing marginal cost $c^{d}$, and increasing in both $c^{d}$ and $\sigma_{T}^{2}$. Also, $\partial \Phi(m) / \partial c^{d}>1$.

Suppose $n=2 m$. Then it is straightforward that $\Omega(m)>0$ if and only if

$$
c^{a}<c^{d}+\frac{(n+1)^{2}(1+\beta) \sigma_{T}^{2}}{4 n(m+2)^{2}\left(\mu_{1}+\mu_{2}-2 c^{d}\right)(1-\beta)}=\Phi(m)
$$

It is obvious $\Phi(m)$ is greater than $c^{d}$ and increasing in both $c^{d}$ and $\sigma_{T}^{2}$.

Define the function

$$
\Delta(m):=\sqrt{1-\frac{(n+1)^{2}(1+\beta)(n-2 m) \sigma_{T}^{2}}{n(m+2)^{2}\left(\mu_{1}+\mu_{2}-2 c^{d}\right)^{2}(1-\beta)}}
$$

If $n \neq 2 m$, we can rearrange $\Omega(m)$ as a quadratic function in $\left(c^{a}-c^{d}\right)$, then factorize as below.

$$
\begin{aligned}
\Omega(m)=\frac{2 n(n-2 m)}{(n+1)^{2}(1+\beta)} & \\
\quad & \quad\left(c^{a}-c^{d}-\frac{\mu_{1}+\mu_{2}-2 c^{d}}{2(n-2 m)}[1+\Delta(m)]\right) \\
& \quad \times\left(c^{a}-c^{d}-\frac{\mu_{1}+\mu_{2}-2 c^{d}}{2(n-2 m)}[1-\Delta(m)]\right)
\end{aligned}
$$

Next, suppose $n>2 m$. Then $\Omega(m)>0$ if and only if

$$
c^{a}>c^{d}+\frac{\mu_{1}+\mu_{2}-2 c^{d}}{2(n-2 m)}[1+\Delta(m)]
$$

or

$$
c^{a}<c^{d}+\frac{\mu_{1}+\mu_{2}-2 c^{d}}{2(n-2 m)}[1-\Delta(m)]=\Phi(m)
$$

The first inequality is ruled out as it implies $K_{i}^{a}(m+1)<0$, contradiction. But then the upper bound for $c^{a}$ is greater than $c^{d}$ and increasing in $\sigma_{T}^{2}$.

Suppose $n<2 m$. Then $\Omega(m)>0$ if and only if

$$
c^{a}>c^{d}+\frac{\mu_{1}+\mu_{2}-2 c^{d}}{2(n-2 m)}[1+\Delta(m)]
$$

and

$$
c^{a}<c^{d}+\frac{\mu_{1}+\mu_{2}-2 c^{d}}{2(n-2 m)}[1-\Delta(m)]=\Phi(m)
$$

The second inequality implies $c^{a}<c^{d}$. But then $\mu_{1}+\mu_{2}+$ $2(n-2 m-1) c^{d}>\mu_{1}+\mu_{2}+(n-2 m-2) c^{d}-(n-2 m) c^{a}$, the latter being positive from the proof of Proposition 3. This in turn implies the first inequality has a negative RHS, and hence is always satisfied. But then, again, the upper bound for $c^{a}$ is greater than $c^{d}$ and increasing in $\sigma_{T}^{2}$.

To complete the proof, observe that for $n \neq 2 m$,

$$
\frac{\partial \Phi\left(m, c^{d}, \sigma_{T}^{2}\right)}{\partial c^{d}}=1+\frac{(1-\Delta) \Delta}{n-2 m}
$$

Because $(1-\Delta)$ and $(n-2 m)$ have the same sign, it is easy to verify that the above is greater than unity whether $n>2 m$ or $n<2 m$.

Once we take demand uncertainty into account, the cost disadvantage of additive manufacturing technology might be outweighed by its flexibility advantage, which is unsurprisingly increasing in demand uncertainty. It is also intuitive why a higher cost of dedicated technology leads to higher tolerance for the cost of additive technology. The interesting part is that in the presence of demand uncertainty, the rate of change of such tolerance with respect to the cost of dedicated technology is more than one-to-one.

\section{CONCLUSIONS}

We have extended the 2-player technology investment game presented in [57] to n-player with a view to adapt their model to accommodate AMs penetration rate in various industries outlined in Gartner hype cycle. Using our extended model, we characterised the market conditions under which it is profitable for a firm to switch to AM technology. We find that the market size effect, substitutability effect, and stochastic effect identified in [57] remain valid in our generalisation. Moreover, in a n-player scenario, if a dedicated firm does not switch to AM technology now, our model suggests that its switching profit will only be lower in the future if other dedicated firms make the switch first. Furthermore, the conventional wisdom of choosing between DM and AM technology stands under the implicit assumption of zero demand uncertainty. However, once we take demand uncertainty into account in our extended model, we find that the cost disadvantage of AM technology might be outweighed by its flexibility advantage, which is increasing in demand uncertainty.

The model investigated in our analysis has certain limitations nonetheless, as it is only the first step in developing an industrially acceptable decision support tool, which will pave the way for a future empirical study. In order to enrich the theoretical understanding of the economic incentive behind the technology investment decision, the scenario of decreasing marginal cost will be examined in future extension to verify the robustness of our results with respect to changes in the cost structure. This extension and further analysis is being carried out in line with the interviews of machine vendors as well as the end-users which have been completed.

\section{REFERENCES}

[1] H. L. Corrła and N. Slack, Framework to analyse flexibility and unplanned change in manufacturing systems, Comput. Integr. Manuf. Syst., vol. 9, no. 1, pp. 5764, Feb. 1996.

[2] D. McFarlane, G. Frizelle, and L. Bongaerts, Disturbance measurement in manufacturing production systems, Proc. ASI June Brem., 1998.

[3] J. George Stalk, TimeThe Next Source of Competitive Advantage, Harvard Business Review, 01-Jul-1988. [Online].

[4] J. L. Bower and T. Hout, Fast-Cycle Capability for Competitive Power, Harvard Business Review, 01-Nov-1988. 
[5] A. Harrison, An Investigation of the Impact of Schedule Stability on Supplier Responsiveness, Int. J. Logist. Manag., vol. 7, no. 1, pp. 8392, Jan. 1996.

[6] R. Suri, How quick response manufacturing takes the wait out, J. Qual. Particip. Cincinnati, vol. 22, no. 3, pp. 4649, Jun. 1999.

[7] M. Holweg, The three dimensions of responsiveness, Int. J. Oper. Prod. Manag., vol. 25, no. 7, pp. 603622, Jul. 2005

[8] A. Puchkova, D. C. McFarlane, R. Srinivasan, and A. Thorne, Optimal Location and Size of Buffers for Disruption-Tolerant Production Operations, EJOR.

[9] D. McFarlane, R. Srinivasan, A. Puchkova, A. Thorne, and A. Brintrup, A Maturity Framework for Operational Resilience and Its Application to Production Control, in Service Orientation in Holonic and Multi-Agen Manufacturing, Springer, Cham, 2018, pp. 5162.

[10] Y. Sheffi and J. Rice James, A Supply Chain View of the Resilient Enterprise, MIT Sloan Manag. Rev., vol. 47, Sep. 2005.

[11] J. Rice James and F. Caniato, Building a secure and resilient supply network, Supply Chain Manag. Rev., vol. 7, pp. 2230, Jan. 2003.

[12] Y. P. Gupta and S. Goyal, Flexibility of manufacturing systems: Concepts and measurements, Eur. J. Oper. Res., vol. 43, no. 2, pp. 119135, Nov. 1989.

[13] D. Gerwin, Manufacturing Flexibility: A Strategic Perspective, Manag. Sci., vol. 39, no. 4, pp. 395410, 1993

[14] D. M. Zelenovic, Flexibilitya condition for effective production systems, Int. J. Prod. Res., vol. 20, no. 3, pp. 319337, May 1982

[15] R. H. Hayes and S. C. Wheelwright, Restoring our Competitive Edge: Competing Through Manufacturing, Jan. 1984.

[16] N. Slack, The Manufacturing Advantage: Achieving Competitive Manufacturing Operations. Mercury, 1991.

[17] D. M. Upton, The Management of Manufacturing Flexibility, Calif. Manage. Rev., vol. 36, no. 2, pp. 7289, Jan. 1994.

[18] M.-H. Chang, Flexible Manufacturing, Uncertain Consumer Tastes, and Strategic Entry Deterrence, J. Ind. Econ., vol. 41, no. 1, pp. 7790, 1993.

[19] B. Lowson, R. King, and A. Hunter, Quick response: managing the supply chain to meet consumer demand. Chichester: Wiley, 1999.

[20] J. Matson and D. McFarlane, Tools for assessing the responsiveness of existing production operations, 1998.

[21] A. K. Sethi and S. P. Sethi, Flexibility in manufacturing: A survey, Int. J. Flex. Manuf. Syst., vol. 2, no. 4, pp. 289328, Jul. 1990.

[22] J. P. Womack, D. T. Jones, and D. Roos, The Machine That Changed the World. [Online].

[23] J. H. Gilmore and B. J. Pine, The four faces of mass customization, Harv. Bus. Rev., vol. 75, no. 1, pp. 91101, Feb. 1997.

[24] J. Lampel and H. Mintzberg, Customizing Customization, Social Science Research Network, Rochester, NY, SSRN Scholarly Paper ID 1862677, 1996.

[25] B. Berman, [3DP Tech Comparison and III phase Evolution] 3-D printing: The new industrial revolution, Bus. Horiz., vol. 55, no. 2, pp 155162, Mar. 2012.

[26] S. Mellor, L. Hao, and D. Zhang, Additive manufacturing: A framework for implementation, Int. J. Prod. Econ., vol. 149, pp. 194201, Mar. 2014.

[27] Y. Huang, M. C. Leu, J. Mazumder, and A. Donmez, Additive Manufacturing: Current State, Future Potential, Gaps and Needs, and Recommendations, J. Manuf. Sci. Eng., vol. 137, no. 1, pp. 014001-01400110, Feb. 2015.

[28] M. K. Thompson et al., Design for Additive Manufacturing: Trends, opportunities, considerations, and constraints, CIRP Ann. - Manuf. Technol., vol. 65, no. 2, pp. 737760, 2016.

[29] B. P. Conner et al., Making sense of 3-D printing: Creating a map of additive manufacturing products and services, Addit. Manuf., vol. 14, pp. 6476 , Oct. 2014

[30] ASTM, Standard Terminology for Additive Manufacturing Technologies.pdf. American Society for Testing and Materials, Mar-2012.

[31] H. Lipson and M. Kurman, Fabricated: The New World of 3D Printing, Wiley.com. [Online].

[32] Y. Koren, General RMS Characteristics. Comparison with Dedicated and Flexible Systems, in Reconfigurable Manufacturing Systems and Transformable Factories, A. I. Dashchenko, Ed. Berlin, Heidelberg: Springer Berlin Heidelberg, 2006, pp. 2745.

[33] A. Dolgui and J.-M. Proth, Supply Chain Engineering: Useful Methods and Techniques. London: Springer-Verlag, 2010.

[34] C. Weller, R. Kleer, and F. T. Piller, Economic implications of 3D printing: Market structure models in light of additive manufacturing revisited, Int. J. Prod. Econ., vol. 164, pp. 4356, Jun. 2015.
[35] P. Reeves and D. Mendis, The Current Status and Impact of 3D Printing Within the Industrial Sector Study II, Mar. 2015

[36] T. Wohlers, Wohlers Report, 2015, Wohlers Associates, 2015.

[37] N. Guo and M. C. Leu, Additive manufacturing: technology, applications and research needs, Front. Mech. Eng., vol. 8, no. 3, pp. 215243, May 2013

[38] Y. Huang, M. C. Leu, J. Mazumder, and A. Donmez, Additive Manufacturing: Current State, Future Potential, Gaps and Needs, and Recommendations, J. Manuf. Sci. Eng., vol. 137, no. 1, pp. 014001014001 Feb. 2015.

[39] P. Milgrom and J. Roberts, The Economics of Modern Manufacturing: Technology, Strategy, and Organization, Am. Econ. Rev., vol. 80, no. 3 , pp. 51128,1990

[40] N. Hopkinson and P. M. Dickens, Analysis of rapid manufacturingusing layer manufacturing processes for production, 2003.

[41] M. Ruffo, C. Tuck, and R. Hague, Cost estimation for rapid manufacturing - laser sintering production for low to medium volumes, Proc. Inst. Mech. Eng. Part B J. Eng. Manuf., vol. 220, no. 9, pp. 14171427, Jan. 2006.

[42] D. S. Thomas and S. W. Gilbert, Costs and Cost Effectiveness of Additive Manufacturing, National Institute of Standards and Technology, NIST SP 1176, Dec. 2014

[43] E. Atzeni and A. Salmi, Economics of additive manufacturing for endusable metal parts, Int. J. Adv. Manuf. Technol., vol. 62, no. 9, pp. 11471155, Oct. 2012

[44] B. Eaton and N. Schmitt, Flexible Manufacturing and Market Structure Am. Econ. Rev., vol. 84, no. 4, pp. 87588, 1994.

[45] G. Norman and J.-F. Thisse, Technology Choice and Market Structure: strategic aspects of flexible manufacturing, J. Ind. Econ., vol. 47, no. 3, pp. 345372, Sep. 1999.

[46] C. H. Fine and R. M. Freund, Optimal Investment in Product-Flexible Manufacturing Capacity, Manag. Sci., vol. 36, no. 4, pp. 449466, Apr. 1990.

[47] W. C. Jordan and S. C. Graves, Principles on the Benefits of Manufacturing Process Flexibility, Manag. Sci., vol. 41, no. 4, pp. 577594, Apr. 1995.

[48] J. A. Van Mieghem, Investment Strategies for Flexible Resources, Manag. Sci., vol. 44, no. 8, pp. 10711078, Aug. 1998.

[49] J. M. Harrison and J. A. Van Mieghem, Multi-resource investment strategies: Operational hedging under demand uncertainty, Eur. J. Oper. Res., vol. 113, no. 1, pp. 1729, Feb. 1999.

[50] S. Netessine, G. Dobson, and R. A. Shumsky, Flexible Service Capacity: Optimal Investment and the Impact of Demand Correlation, Oper. Res. vol. 50 , no. 2 , pp. 375388 , Apr. 2002

[51] B. Tomlin and Y. Wang, On the Value of Mix Flexibility and Dual Sourcing in Unreliable Newsvendor Networks, Manuf. Serv. Oper. Manag., vol. 7, no. 1, pp. 3757, Jan. 2005.

[52] J. Chod and N. Rudi, Resource Flexibility with Responsive Pricing, Oper. Res., vol. 53, no. 3, pp. 532548, Jun. 2005

[53] C. Gaimon, Dynamic Game Results of the Acquisition of New Technology, Oper. Res., vol. 37, no. 3, pp. 410425, Jun. 1989.

[54] C. H. Fine and S. Pappu, Flexible manufacturing technology and product-market competition, Massachusetts Institute of Technology (MIT), Sloan School of Management, 313590., 1990.

[55] L.-H. Roeller and M. M. Tombak, Competition and investment in flexible technologies, Manag. Sci., vol. 39, no. 1, pp. 107114, 1993.

[56] L.-H. Rller and M. M. Tombak, Competition and Investment in Flexible Technologies, Manag. Sci., vol. 39, no. 1, pp. 107114, 1993.

[57] M. Goyal and S. Netessine, Strategic technology choice and capacity investment under demand uncertainty, Manag. Sci., vol. 53, no. 2, pp 192207, 2007.

[58] Boonman, H. J., Hagspiel, V. and Kort, P. M. Dedicated vs product flexible production technology: strategic capacity investment choice. European Journal of Operational Research, 244 (1). 2015.

[59] He, P., Ding, H. and Hua, Z. Strategic choice of flexible production technology using game theory approach. Robotics and Computer-Integrated Manufacturing, 28 (3). 2012. 